\title{
DISTRIBUCIÓN, MORFOLOGÍA Y REPRODUCCIÓN DEL MURCIÉLAGO RAYADO DE OREJAS AMARILLAS VAMPYRISCUS NYMPHAEA (MAMMALIA: CHIROPTERA) EN COLOMBIA
}

\author{
Miguel E. RODRÍGUEZ-POSADA ${ }^{\mathbf{1}}$ \& HÉCTOR E. RAMÍREZ-CHAVES ${ }^{2}$ \\ ${ }^{1}$ Grupo de investigación en conservación y manejo de vida silvestre, Universidad Nacional de \\ Colombia. Dirección correspondencia: Calle 162 \# 54-09 torre 1, apartamento 404, Senderos del \\ Carmel 2. Bogotá D. C., Colombia. <migrodriguezp@yahoo.com> \\ ${ }^{2}$ Erasmus Mundus Master Programme in Evolutionary Biology: Ludwig Maximilians University of \\ Munich, Germany y University of Groningen, The Netherlands. < hera.chaves@gmail.com>
}

Rodríguez-Posada, M. E. \& H. E. Ramírez-Chaves. 2012. Distribución, morfología y reproducción del murciélago rayado de orejas amarillas Vampyriscus nymphaea (Mammalia: Chiroptera) en Colombia. Acta Zoológica Mexicana (n. s.), 28(2): 341-352.

RESUMEN. Presentamos información sobre la distribución geográfica, morfología y reproducción de Vampyriscus nymphaea en Colombia, basándonos en la revisión de especímenes museológicos de colecciones colombianas. Previamente la distribución de $V$. nymphaea en Colombia se consideraba restringida a las tierras bajas al occidente de la cordillera Occidental en la región Pacífico; en este trabajo confirmamos la presencia de esta especie en la región Caribe y en el nororiente de la cordillera Occidental de los Andes colombianos en el Bajo Río Cauca. La morfología externa y craneana de la especie fue homogénea y el análisis de dimorfismo sexual secundario de las poblaciones de la región Pacífico no mostró diferencias significativas, sin embargo la longitud de la tibia y la profundidad de la caja craneana son proporcionalmente mayores en los machos y el ancho zigomático en las hembras. Respecto a la biología reproductiva sugerimos que las poblaciones de $V$. nymphaea presentan un patrón poliéstrico sin picos definidos a lo largo del año.

Palabras clave: Andes, Chocó biogeográfico, morfología, morfometría, región Caribe, valles interandinos.

Rodríguez-Posada, M. E. \& H. E. Ramírez-Chaves. 2012. Distribution, morphology and reproduction of the striped yellow-eared bat Vampyriscus nymphaea (Mammalia: Chiroptera) in Colombia. Acta Zoológica Mexicana (n. s.), 28(2): 341-352.

ABSTRACT. We present information related on the distribution, morphology and reproductive biology of Vampyriscus nymphaea in Colombia, based in the analysis of voucher specimens housed in Colombia collections. The known distribution of $V$. nymphaea in Colombia previously restricted to the western lowlands of the Cordillera Occidental in the Pacific region is extended to the Caribbean region and to

Recibido: 11/04/2011; aceptado: 19/01/2012. 
the north-eastern part of the Cordillera Occidental, lower Cauca River Basin of Colombia. Both external and the skull morphology of the species were homogeneous; the analysis of the secondary sexual dimorphism among specimens from the Pacific area do not show significant differences, although, the length of the tibia and the deep of the skull were proportionally larger in males, and the zygomatic width in females. Concerning to the reproductive biology, we suggest that the populations of $V$. nymphaea present a poliestric pattern along the year but without defined peaks.

Key words: Andes, Biogeographic Chocó, Caribean region, interandean valleys, morphology, morphometrics.

\section{INTRODUCCIÓN}

El género Vampyriscus Thomas, 1900 contiene tres especies: Vampyriscus bidens (Dobson, 1878), Vampyriscus brocki (Peterson, 1968) y Vampyriscus nymphaea (Thomas, 1909). Estas especies estaban incluidas en el género Vampyressa junto con Vampyressa melissa Thomas, 1926, Vampyressa pusilla (Wagner, 1843) y Vampyressa thyone Thomas 1909 (Simmons 2005), donde V. nymphaea y V. brocki conformaban el subgénero Metavampyressa Peterson 1968. Sin embargo, Porter \& Baker (2004) reconocieron Vampyriscus como un linaje independiente de Vampyressa y lo elevaron a categoría de género incluyendo a $V$. bidens y $V$. brocki. Hoofer \& Baker (2006) encontraron que Vampyriscus está más relacionado filogenéticamente con Chiroderma que con Vampyressa e incluyeron a $V$. nymphaea dentro del género, tratamiento que ha sido aceptado por otros autores (Arroyo-Cabrales 2008).

En Colombia habitan las tres especies del género Vampyriscus: V. bidens cuya distribución comprende la vertiente oriental de los Andes extendiéndose a través de la Orinoquía y la Amazonía (Lee et al. 2001); V. brocki presente en la Amazonía suroccidental, en el sur del departamento del Amazonas (Alberico et al. 2000) y V. nymphaea, que se distribuye en la región Pacífico, al occidente de los Andes entre los 0 y 1900 m de altitud, en los departamentos de Antioquia, Chocó, Valle del Cauca y Nariño (Alberico et al. 2000, Muñoz 2001, Arroyo-Cabrales 2008). Hasta el momento los registros en la literatura en otras regiones, corresponden a posibles malas identificaciones o reportes sin especímenes de referencia (Arroyo-Cabrales 2008).

El murciélago rayado de orejas amarillas es considerado una especie rara o poco común (LaVal \& Rodríguez-H 2002, Rodríguez-Herrera \& Tschapka 2005). Su historia natural es poco conocida (Tavares et al. 2008), algunos aspectos sobre su ecología son resumidos por Arroyo-Cabrales (2008), pero el conocimiento sobre su distribución, variación intraespecífica y reproducción es aún incompleto. En el presente trabajo revisamos la distribución de $V$. nymphaea en Colombia con base en especímenes de museo y registros confirmados en la literatura. Adicionalmente, analizamos la variación morfológica en el país con énfasis en la variación sexual secundaria en las poblaciones del Pacífico y finalmente compilamos la información asociada a los especímenes que examinamos para aportar información sobre la biología reproductiva de la especie. 


\section{MATERIALES Y MÉTODOS}

Los datos de la distribución en Colombia de V. nymphaea fueron obtenidos a partir de los especímenes depositados en las colecciones mastozoológicas del país: Instituto de Ciencias Naturales de la Universidad Nacional de Colombia (ICN), Universidad del Valle (UV), Instituto de Investigación en Recursos Biológicos Alexander von Humboldt (IAvH) y la Colección Teriológica de la Universidad de Antioquia (CTUA, incluye ejemplares del Museo de la Universidad de Antioquia MUA). Verificamos la identificación taxonómica de todos los especímenes disponibles (Anexo 1) con base en los trabajos de Thomas (1909), Goodwin (1963), Peterson (1968) y ArroyoCabrales (2008) y anotamos sus localidades. Para complementar ésta información incluimos los datos de reportes en la literatura con localidad precisa y especímenes de referencia existentes (Thomas 1909, Goodwin 1963, Asprilla-Aguilar et al. 2007, Ramírez-Chaves \& Pérez 2011). Las localidades fueron georeferenciadas a partir de información suministrada en las etiquetas de los especímenes de museo y mediante el uso de gaceteros (Paynter 1997), posteriormente generamos un mapa con los puntos de distribución de la especie en el país (Fig. 1).

Para estudiar la variación morfológica de la especie en Colombia, hicimos observaciones detalladas de 77 especímenes y tomamos 12 medidas craneanas, dos mandibulares y dos externas siguiendo los criterios métricos de Rodríguez-Posada \& Sánchez-Palomino (2009): Longitud mayor del cráneo (LMC), longitud cóndiloincisivo (LCI), anchura zigomática (AZ), anchura postorbital (AP), anchura de la caja cerebral (ACC), anchura mastoidea (AM), altura de la caja cerebral (PCC), longitud de la hilera dental maxilar (LHDMax), longitud del paladar (LPal), longitud postpalatal (LPPal), anchura maxilar (AMax), ancho entre los caninos (C-C), longitud del dentario (LDen), hilera dental inferior (HDI), longitud del antebrazo (LA), longitud de la tibia (Lt).

Para el análisis de la variación sexual secundaria, comparamos 15 hembras y 15 machos provenientes de poblaciones de la región Pacífico. Utilizamos 11 variables craneanas y las longitudes del antebrazo y la tibia. Empleamos los datos completos con sus valores originales, probando previamente que cumplieran los supuestos de aleatoriedad (prueba de corridas por arriba y por debajo), normalidad (prueba de Shapiro-Wilk) y homogeneidad de varianzas (pruebas de Barlett y de Levene). La longitud postpalatal fue omitida de los análisis porque no cumplió con los supuestos de normalidad y homogeneidad de varianza. Para la comparación general entre los grupos utilizamos un análisis multivariado de varianza (MANOVA) con la prueba Lambda de Wilk. Adicionalmente hicimos comparaciones entre cada variable empleada en el análisis, a través de análisis univariados de varianza (ANOVA). Los análisis fueron corridos en el programa PAST (PAlaentological STatistics) v. 2.07 (Hammer et al. 2001), teniendo en cuenta un nivel de significancia estadística de $\alpha=0,05$. 


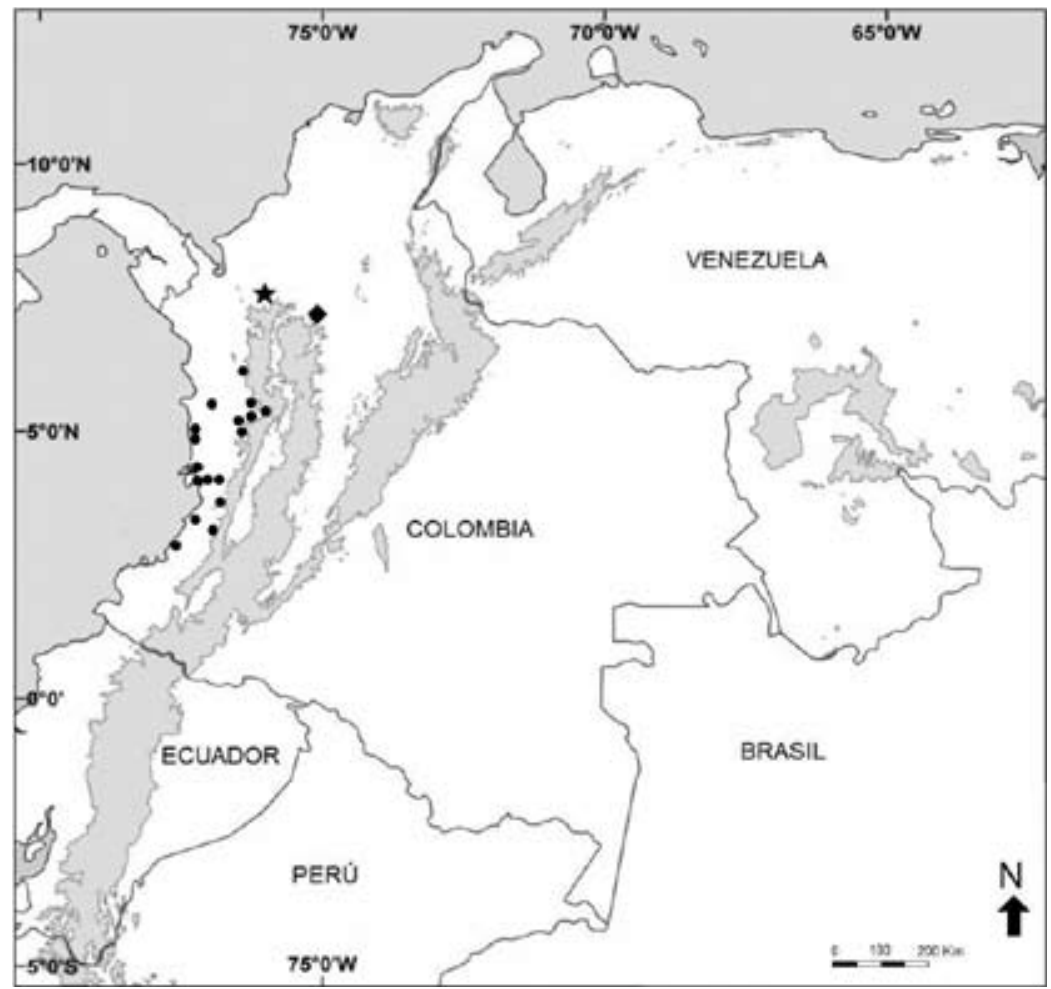

Figura 1. Localidades de registro de Vampyriscus nymphaea en Colombia (Anexo 1). Las localidades de registro fuera de la región Pacífico están denotadas con una estrella (ICN 19917: Tierralta, departamento de Córdoba) y un rombo (IAvH 976: Zaragoza, La Tirana, departamento de Antioquia).

Finalmente, adicionamos información sobre la reproducción de la especie con base en los datos de las etiquetas de los especímenes hembras que revisamos. Las categorías reproductivas que consideramos fueron gestantes, lactantes y no reproductivas.

\section{RESULTADOS}

La mayoría (22) de las 24 localidades de presencia confirmada de $V$. nymphaea en Colombia (Anexo 1) están ubicadas en la región Pacífico. Dos especímenes provienen de dos localidades por fuera de la distribución conocida para la especie, siendo los primeros registros confirmados de V. nymphaea para la región Caribe (ICN 19917) y el Bajo Río Cauca en la región Andina (IAvH 976) (Fig. 1).

Los especímenes examinados coinciden en todas las características morfológicas y morfométricas conocidas para V. nymphaea (Thomas 1909, Goodwin 1963, Peter- 
son 1968, Arroyo-Cabrales 2008). El pelaje dorsal es de color gris humo ligeramente escarchado y todos los especímenes examinados presentan una línea longitudinal difusa o poco visible en el dorso. El rostro presenta cuatro líneas blancas bien marcadas y conspicuas, las supraorbitales se extienden sobre la cabeza hasta atrás de la orejas. Los bordes de las orejas y de la hoja nasal son de color amarillo claro. El trago es completamente amarillo, liso, ligeramente aserrado en el borde externo y sin pelos en la base. El plagiopatagio está unido a las extremidades inferiores en el metatarso. La membrana interfemoral es ancha con el borde libre liso y desnudo a excepción de una pequeña orla de pelo hacia la parte media.

Todos los especímenes examinados presentaron una morfología craneana homogénea, a excepción de un espécimen subadulto proveniente del Chocó (ICN 16165) que presenta el borde posterior del paladar con una hendidura en forma de "V", mientras que los otros especímenes lo presentan completamente recto. La dentición se caracterizó por presentar dos pares de incisivos superiores e inferiores, los superiores centrales son tres veces más largos que los laterales, pincelados, convergiendo y uniéndose hacia el tercio distal, con una pequeña bilobulación asimétrica en la punta (Fig. 2). Los caninos son cónicos y simples, ligeramente curvados hacia atrás. Dos pares de premolares superiores e inferiores, el premolar superior anterior es triangular y sin cúspides accesorias, su tamaño es pequeño (1/3 ó menos) en relación al canino y al premolar posterior superior, este último es cónico y delgado, presenta una pequeña cúspide posterior ubicada sobre el cíngulo y un talón desarrollado hacia la región labial. Dos pares de molares superiores e inferiores, los molares superiores son más anchos que largos, con talones amplios y bien desarrollados. El molar anterior superior presenta en la región lingual dos cúspides altas, la posterior con una pequeña cúspide accesoria; y en la región labial presenta solo una cúspide baja. El molar superior posterior presenta en la región labial dos cúspides de las cuales la anterior es alta y la posterior baja; en la región lingual presenta una cúspide baja. Existe variación en las proporciones de ancho y longitud de los talones de los molares, sin embargo al observar cada diente individualmente y compararlo con su homólogo en otros especímenes se observa que la morfología general de cada diente se conserva.

El análisis de dimorfismo sexual secundario no mostró diferencias significativas entre machos y hembras $\left(\mathrm{F}_{13}, 16=2.19 ; \mathrm{P}=0.07\right)$. Los análisis variable por variable, mostraron que machos y hembras se sobreponen en todas las medidas comparadas, sin embargo la longitud de la tibia $\left(\mathrm{F}_{1},{ }_{28}=13.28, \mathrm{P}=0.001\right)$, la anchura zigomática $\left(\mathrm{F}_{1}, 28=8.51, \mathrm{P}=0.007\right)$ y la profundidad de la caja craneana $\left(\mathrm{F}_{1}, 28=11.75, \mathrm{P}=\right.$ $0.002)$ presentan diferencias significativas entre los dos sexos. Los machos presentan tibias más largas y cajas craneanas más altas, mientras las hembras presentan arcos zigomáticos más anchos (Cuadro 1). 


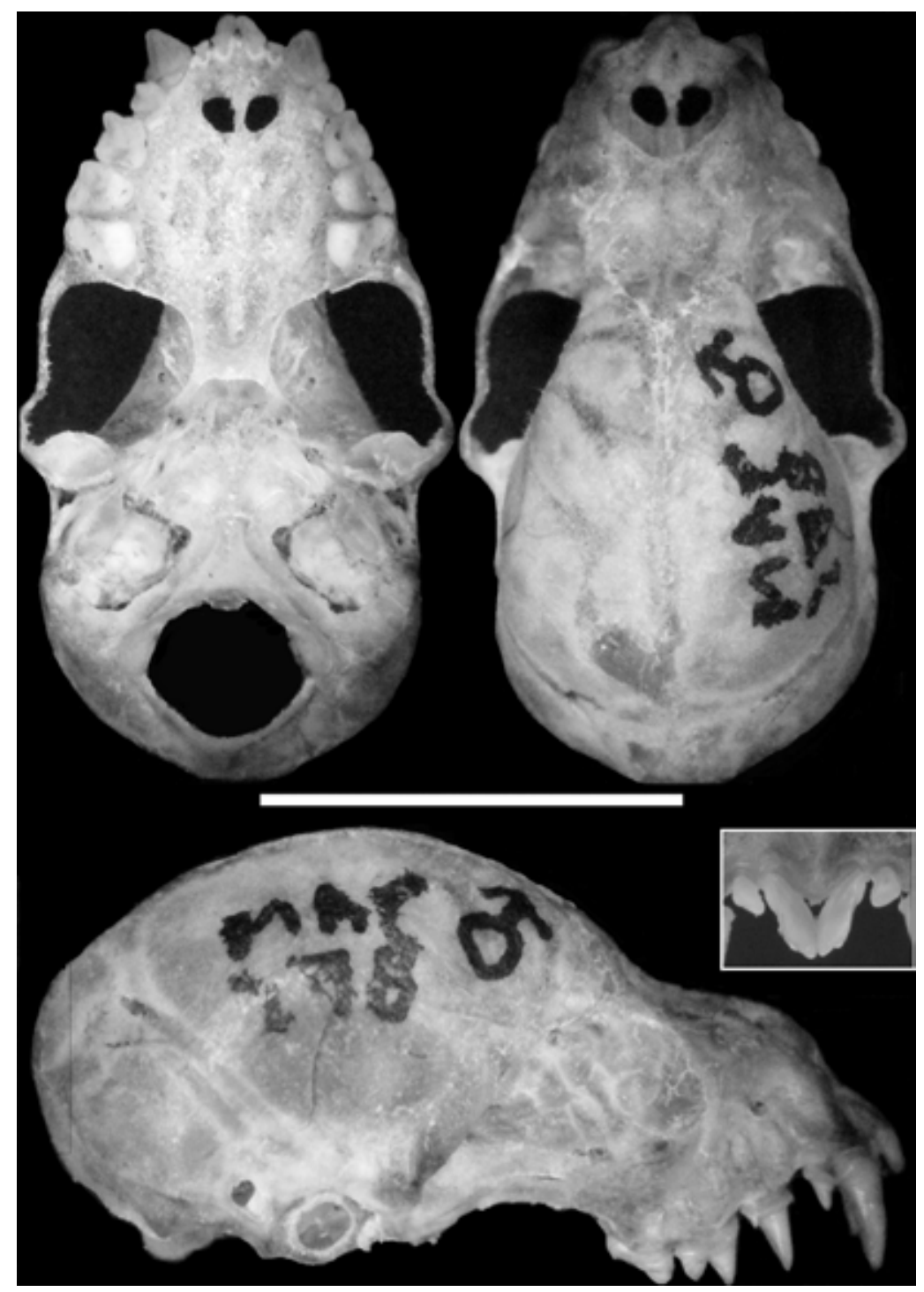

Figura 2. Cráneo de Vampyriscus nymphaea ICN 19917. Vista ventral arriba izquierda. Vista dorsal arriba derecha. Vista lateral abajo. Incisivos superiores en el recuadro. Barra de escala $=10 \mathrm{~mm}$.

En la recopilación de datos reproductivos encontramos hembras inactivas en el mes de mayo (dos individuos); lactantes en los meses de febrero (uno), marzo (tres), mayo (dos) y agosto (tres); y gestantes en enero (seis), febrero (uno), marzo (uno), agosto (uno) y diciembre (dos). 
Cuadro 1. Medidas comparadas entre machos y hembras de Vampyriscus nymphaea en la región Pacífico de Colombia. Los acrónimos de las medidas corresponden a las expuestas en los métodos. Media \pm desviación estándar en la celda superior y rango de las medidas en la celda inferior y entre paréntesis. $\mathrm{N}=15$ para cada sexo.

\begin{tabular}{|c|c|c|c|c|c|c|}
\hline \multirow[b]{2}{*}{ LA } & \multicolumn{3}{|c|}{ Hembras } & \multicolumn{3}{|c|}{ Machos } \\
\hline & 36.86 & \pm & 0.69 & 36.73 & \pm & 0.99 \\
\hline & \multicolumn{3}{|c|}{ (35.37-37.81) } & \multicolumn{3}{|c|}{ (34.84-39.03) } \\
\hline \multirow[t]{2}{*}{ Tibia } & 12.77 & \pm & 1.03 & 14.07 & \pm & 0.92 \\
\hline & \multicolumn{3}{|c|}{$(11.00-14.46)$} & \multicolumn{3}{|c|}{ (11.58-15.98) } \\
\hline \multirow[t]{2}{*}{ LMC } & 20.83 & \pm & 0.28 & 20.80 & \pm & 0.36 \\
\hline & \multicolumn{3}{|c|}{ (20.36-21.29) } & \multicolumn{3}{|c|}{$(20.20-21.56)$} \\
\hline \multirow[t]{2}{*}{ LCI } & 18.85 & \pm & 0.34 & 18.70 & \pm & 0.25 \\
\hline & \multicolumn{3}{|c|}{ (18.15-19.37) } & \multicolumn{3}{|c|}{$(18.37-19.2)$} \\
\hline \multirow[t]{2}{*}{$\mathrm{AZ}$} & 12.32 & \pm & 0.21 & 12.09 & \pm & 0.22 \\
\hline & \multicolumn{3}{|c|}{$(11.95-12.75)$} & \multicolumn{3}{|c|}{ (11.64-12.37) } \\
\hline \multirow[t]{2}{*}{ AP } & 4.80 & \pm & 0.18 & 4.78 & \pm & 0.13 \\
\hline & \multicolumn{3}{|c|}{$(4.52-5.06)$} & \multicolumn{3}{|c|}{$(4.63-5.04)$} \\
\hline \multirow[t]{2}{*}{ ACC } & 9.16 & \pm & 0.15 & 9.18 & \pm & 0.18 \\
\hline & \multicolumn{3}{|c|}{ (8.95-9.44) } & \multicolumn{3}{|c|}{$(8.84-9.4)$} \\
\hline \multirow[t]{2}{*}{$\mathrm{AM}$} & 10.46 & \pm & 0.24 & 10.33 & \pm & 0.18 \\
\hline & \multicolumn{3}{|c|}{ (10.12-10.93) } & \multicolumn{3}{|c|}{ (9.91-10.59) } \\
\hline \multirow[t]{2}{*}{ PCC } & 7.90 & \pm & 0.17 & 8.09 & \pm & 0.13 \\
\hline & \multicolumn{3}{|c|}{ (7.69-8.22) } & \multicolumn{3}{|c|}{ (7.81-8.31) } \\
\hline \multirow[t]{2}{*}{ LHDM } & 7.22 & \pm & 0.11 & 7.19 & \pm & 0.10 \\
\hline & \multicolumn{3}{|c|}{ (7.04-7.43) } & \multicolumn{3}{|c|}{$(7.03-7.45)$} \\
\hline LPAL & 9.70 & \pm & 0.44 & 9.43 & \pm & 0.32 \\
\hline & & -10 & & & $1-9$ & \\
\hline AMAX & 8.86 & \pm & 0.21 & 8.76 & \pm & 0.16 \\
\hline & & $1-9$ & & & $7-9$ & \\
\hline ACAN & 4.74 & \pm & 0.14 & 4.73 & \pm & 0.18 \\
\hline & & $4-4$ & & & $1-5$ & \\
\hline
\end{tabular}

\section{DISCUSIÓN}

En general, la distribución geográfica de $V$. nymphaea en Colombia ha sido restringida a la región Pacífico que hace parte del Chocó biogeográfico y se ubica en un área geológicamente reciente de la costa Pacífica de Colombia y Ecuador, caracterizada por tierras bajas y húmedas, con una alta pluviosidad (Gentry 1982). 
Dos localidades de registro de $V$. nymphaea amplían la distribución de la especie a regiones distintas a la región Pacífico. Una de éstas (ICN 19917) está ubicada en la región Caribe que se extiende desde el extremo noroccidental, cerca a la frontera con Panamá, hasta la península de la Guajira y que se caracteriza por presentar bosques secos a ligeramente húmedos (Galeano 1992); sin embargo en el sitio de registro la vegetación corresponde a remanentes de bosque húmedo tropical. La otra localidad (IAvH 976) corresponde a la región del Bajo Río Cauca, que abarca el valle interandino comprendido entre las cordilleras Occidental y Central en el departamento de Antioquia, cuya vegetación también corresponde a bosque húmedo tropical.

Nuestros resultados muestran que aunque la mayoría de registros de $V$. nymphaea en Colombia provienen de la región Pacífico, ésta también habita los ecosistemas de bosque húmedo tropical de la región Caribe que coinciden con los distritos del sector Chocó y los distritos Sinú-San Jorge y Nechí del sector del Magdalena de la Provincia biogeográfica Chocó-Magdalena propuesta por Hernández-Camacho et al. (1992), y que al parecer representan el límite oriental de elementos de biota distribuidos en el Chocó biogeográfico y Centroamérica. Probablemente la especie puede distribuirse hasta las tierras bajas del sector comprendido entre las cuencas de los ríos Sinú, San Jorge, el Bajo Río Cauca e incluso parte de la cuenca media del Río Magdalena en el departamento de Antioquia incluyendo la serranía de San Lucas donde la formación vegetal original corresponde a bosque húmedo tropical. La distribución de la especie puede estar limitada al nororiente por el cinturón árido pericaribeño, especialmente por las sabanas del Caribe y las zonas secas del Magdalena Medio (Hernández-Camacho et al. 1992).

Registros por fuera de las zonas de Colombia mencionadas corresponden a identificaciones erróneas o reportes sin especímenes de referencia, por lo cual descartamos la presencia de la especie en los Andes o en las tierras bajas al oriente de estos (Orinoquía y Amazonía). Previo a nuestro estudio, Cuervo Díaz et al. (1986) habían propuesto que la distribución de $V$. nymphaea en Colombia comprendía la región Pacífico y una zona en el extremo occidental de la región Caribe hasta el río Sinú. Dichos autores no mencionaron especímenes de referencia o citas bibliográficas que permitiera corroborar esta afirmación. Nuestros resultados confirman que la especie se distribuye en dicha región alcanzando hasta la parte norte de la cordillera Central. Por otra parte, Cuartas-Calle \& Muñoz-Arango (2003) mencionaron que $V$. nymphaea se distribuye en los bosques húmedos tropicales al oriente de Antioquia entre los 0 y $1900 \mathrm{~m}$, citan a Alberico et al. (2000) quienes restringen la especie a la región Pacífico y agregan que en la colección del MUA hay depositados especímenes que apoyan su afirmación; sin embargo en dicha colección solo se encuentran dos especímenes de esta especie y proceden del departamento de Chocó (Solari 2010, comunicación personal). 
Otros registros publicados de V. nymphaea por fuera de la región Pacífico en Colombia merecen ser discutidos: Otálora-Ardila (2003) mencionó que la especie habitaba los bosques de roble (Quercus humboldtii) de la vertiente occidental de la cordillera Oriental de Colombia (Charalá, Santander). Este registro se basó en información secundaria (informes de campo) y la autora aclaró que no revisó las identificaciones taxonómicas de los especímenes de referencia que se encuentran depositados en la colección del ICN. Nosotros revisamos todos los especímenes disponibles de $V$. nymphaea del ICN y no encontramos ningún ejemplar procedente de Charalá, por lo que el registro de Otálora-Ardila (2003) debe corresponder a una identificación de campo errónea. Mantilla-Meluk et al. (2009) presentaron un modelo de distribución potencial de $V$. nymphaea en Colombia, que restringe la especie al sur de la Amazonía colombiana; aparentemente este es un error en los títulos de los mapas ya que en el cuadro resumen de la distribución aparece que la distribución de $V$. nymphaea está restringida para la región Pacífico (Mantilla-Meluk et al. 2009). Para el valle medio del Río Magdalena, hay un registro publicado de $V$. nymphaea procedente de Totare (2400 m), departamento del Tolima, depositado en la Colección Zoológica, Universidad del Tolima-Mastozoología CZUT-M (CZUT-M 0737) (Galindo-Espinosa et al. 2011), pero al revisar una imagen del cráneo del espécimen pudimos verificar que correspondía a un Dermanura cf. bogotensis. Finalmente consideramos necesario mencionar los especímenes que pudimos verificar y que están erróneamente identificados como $V$. nymphaea y aparecen así en la base de datos en línea de su correspondiente colección: ICN 9457 (Meta: Fuente de Oro) cuya piel corresponde a un Dermanura sp. y el cráneo a un Platyrrhinus brachycephalus; ICN 15061 (Boyacá: Santa María) que corresponde a un Dermanura cf. bogotensis; e ICN 17325 (Córdoba: Montería) correspondiente a un Platyrrhinus helleri.

En general no observamos diferencias morfológicas craneanas ni externas entre los especímenes examinados de la región Pacífico, y entre éstos con los de la región Caribe. V. nymphaea puede ser diferenciada de otras especies similares en Colombia con base en los caracteres y medidas mencionados en la sección de resultados (Cuadro 1, Fig. 2). Nuestro análisis general de dimorfismo sexual secundario no mostró diferencias entre machos y hembras, sin embargo el valor crítico de significancia es muy bajo y recomendamos hacer análisis con un mayor número de individuos para verificar nuestros resultados y su utilidad para futuros análisis de variación geográfica. También sugerimos presentar análisis de dimorfismo sexual de poblaciones que habiten zonas diferentes a las selvas húmedas de la región Pacífico colombiana.

Como hemos mencionado anteriormente, la historia natural de esta especie es poco conocida (Tavares et al. 2008) y se ha sugerido que su patrón de reproducción corresponde a poliestría bimodal con picos en febrero y agosto (Wilson 1979, Arroyo-Cabrales 2008); Wilson (1979) registró para Colombia hembras preñadas o lactantes en los meses de octubre y agosto, mientras que en Centroamérica en los meses 
de febrero y abril. Nosotros reportamos hembras lactantes o preñadas en los meses de enero, febrero, marzo, mayo, agosto y diciembre. Sugerimos que las poblaciones de $V$. nymphaea presentan un patrón poliéstrico, pero sin picos definidos a lo largo del año.

Finalmente recomendamos a los investigadores verificar las identificaciones de los datos de distribución extraídos de las bases de datos de colecciones científicas, así como revisar críticamente la información publicada.

Agradecimientos. Queremos agradecer a las personas que nos facilitaron examinar los especímenes de las colecciones revisadas: Yaneth Muñoz-Saba y Hugo López-Arévalo (ICN), Oscar Murillo (UV), José Enrique Castillo (IAvH), Sergio Solari (CTUA). Jaider Manuel Peña de la Colección Zoológica de la Universidad del Tolima, tomó y facilitó las imágenes de ejemplar CZUT M 0737. Agradecemos a Julio Betancur por la colaboración en la obtención de las imágenes de los cráneos. Leison Palacios Mosquera y Weimar A. Pérez aportaron información importante para el desarrollo del manuscrito. Andrés Felipe Suárez y tres evaluadores anónimos hicieron comentarios editoriales valiosos al manuscrito.

\section{LITERATURA CITADA}

Alberico, M., A. Cadena, J. Hernández-Camacho \& Y. Muñoz-Saba. 2000. Mamíferos (Synapsida: Theria) de Colombia. Biota Colombiana, 1: 43-75.

Arroyo-Cabrales J. 2008. Vampyriscus O. Thomas, 1900, pp. 350-355. In: A. L. Gardner (Ed.). Mammals of South America, Volume 1: Marsupials, Xenarthrans, Shrews, and Bats. The University of Chicago Press, Chicago.

Asprilla-Aguilar, A. A., H. Mantilla-Meluk \& A. M. Jiménez-Ortega. 2007. Analysis of the non-hematophagous bat species captured within the plan of eradication of Desmodus rotundus (I. Geoffroyi, 1810) in the Colombian Biogeographic Chocó. Revista Institucional Universidad Tecnológica del Chocó D. L. C., 26: 42-48.

Cuartas-Calle, C.A \& J. Muñoz-Arango. 2003. Lista de los mamíferos (Mammalia: Theria) del departamento de Antioquia, Colombia. Biota Colombiana, 4: 65-78

Cuervo Díaz, A., J. Hernández-Camacho \& A. Cadena G. 1986. Lista actualizada de los mamíferos de Colombia. Anotaciones sobre su distribución. Caldasia, 15: 471-502.

Galeano, G. 1992. Patrones de distribución de las palmas de Colombia. Bulletin de l'Institut Français d'Etudes Andines, 21: 599-607.

Galindo-Espinosa, E. Y., K. A. Gutierrez-Díaz \& G. Reinoso-Flórez. 2010. Lista de los quirópteros del departamento del Tolima, Colombia. Biota Colombiana, 11: 107-116.

Gentry, G. H. 1982. Neotropical floristic diversity: Phytogeographical connections between Central and South America, Pleistocene climatic fluctuations, or an accident of the Andean orogeny? Annals of the Missouri Botanical Garden, 69: 557-593.

Goodwin, G. G. 1963. American bats of the genus Vampyressa, with description of a new species. American Museum Novitates, 2125: 1-24

Hammer, O. D., A. T. Harper \& P. D. Ryan. 2001. PAST: PAleontological STatistics software package for education and data analysis. Palaentologia Electronica 4:1-9. http://folk.uio.no/ohammer/ past.

Hernández-Camacho, J. I., A. Hurtado-Guerra, R. Ortiz Quijano \& T. Walschburger. 1992. Unidades biogeográficas de Colombia, pp. 105-152. In: G. Haffer (Ed.). La diversidad biológica en 
Iberoamérica I. Acta Zoológica Mexicana, Volumen especial. Programa Iberoamericano de Ciencia y Tecnología para el Desarrollo, Instituto de Ecología, A. C. Xalapa, Veracruz, México.

Hoofer, S. R. \& R. J. Baker. 2006. Molecular systematics of Vampyressine bats (Phyllostomidae: Stenodermatinae) with comparison of direct and indirect surveys of mitochondrial DNA variation. Molecular Phylogenetics and Evolution, 39: 424-438.

Laval, R. K. \& B. Rodríguez-H. 2002. Murciélagos de Costa Rica. Editorial INBio. Santo Domingo de Heredia, Costa Rica, 320 pp.

Lee, T. E. Jr., J. B. Scott \& M. M. Marcum. 2001. Vampyressa bidens. Mammalian Species, 684: $1-3$.

Mantilla-Meluk, H., A .M. Jiménez-Ortega \& R. J. Baker. 2009. Phyllostomid Bats of Colombia: Annotated checklist, distribution, and biogeography. Special Publications, Museum of Texas Tech University, 56: 1-37.

Muñoz J. 2001. Los Murciélagos de Colombia. Sistemática, distribución, descripción, historia natural y ecología. Editorial Universidad de Antioquia, Medellín, 391 pp.

Otálora Ardila, A. 2003. Mamíferos de los bosques de roble. Acta Biológica Colombiana 8: 57-71.

Paynter, R. A., Jr. 1997. Ornithological Gazetteer of Colombia, second edition. Museum of Comparative Zoology, Cambridge, Massachusetts, USA, 537 pp.

Peterson, R. L. 1968. A new bat of the genus Vampyressa from Guyana, South America with brief systematic review of the genus. Life Sciences Occasional Papers, Royal Ontario Museum, 73: 1-17.

Porter, C. A., \& R. J. Baker. 2004. Systematics of Vampyressa and related genera of phyllostomid bats as determined by cytochrome-b sequences. Journal of Mammalogy, 85: 126-132.

Ramírez-Chaves, H. E. \& W. A. Pérez. 2011. “2010”. Mamíferos (Mammalia: Theria) del departamento del Cauca, Colombia. Biota Colombiana, 11: 141-171.

Rodríguez-Herrera, B. \& M. Tschapka. 2005. Tent use by Vampyriscus nymphaea (Chiroptera: Phyllostomidae) in Cecropia insignis (Moraceae) in Costa Rica. Acta Chiropterologica, 7: 171-174.

Rodríguez-Posada, M. E. \& P. Sánchez-Palomino. 2009. Taxonomía del género Phyllostomus (Chiroptera: Phyllostomidae) en Colombia. Mastozoología Neotropical, 16:153-168.

Simmons, N. B. 2005. Order Chiroptera, pp 312-529. In: D. E. Wilson y D. M. Reeder (Eds.). Mammal species of the world: a taxonomic and geographic reference. The Johns Hopkins University Press. Baltimore.

Tavares, V., A. Muñoz, B. Rodriguez \& J. Arroyo-Cabrales. 2008. Vampyressa nymphaea. En: IUCN 2009. IUCN Red List of Threatened Species. Version 2009.2. <www.iucnredlist.org>. Downloaded on 20 January 2010.

Thomas, O. 1909. Notes on some South-American mammals, with descriptions of new species. Annals and Magazine of Natural History 8: 230-232.

Wilson, D. E. 1979. Reproductive patterns, pp. 317-378. In: R. J. Baker, J. K. Jones Jr. y D. C. Carter (Eds.). Biology of bats of the New World Family Phyllostomatidae. Part III. Special Publication, Museum of Texas Tech University, 16: 1-441. 


\begin{abstract}
ANEXO 1
Localidades de registro de Vampyriscus nymphaea en Colombia, coordenadas geográficas entre paréntesis seguidas por el número de colección de los especímenes examinados o la fuente bibliográfica. Los especímenes denotados con asterisco (*) fueron los incluidos en el análisis de dimorfismo sexual secundario.
\end{abstract}

1. Departamento de Antioquia, Zaragoza, La Tirana, $25 \mathrm{Km}$ Sur $+22 \mathrm{Km}$ occidente de Zaragoza (07²9’39” N, 7452'16” W; 64 m) IAvH 976; 2. Departamento del Cauca, Timbiquí, quebrada Guangüi, $0.5 \mathrm{Km}$ norte del río Patía, cuenca alta del rio Saija $\left(02^{\circ} 52^{\prime} \mathrm{N}, 77^{\circ} 41^{\prime} \mathrm{W}\right)$ Ramírez-Chaves \& Pérez (2011); 3. Departamento de Chocó, Novitá, río San Juan (0457`21” N, 76³6’59” W; 46 m)

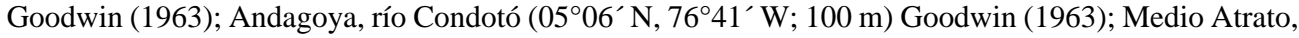

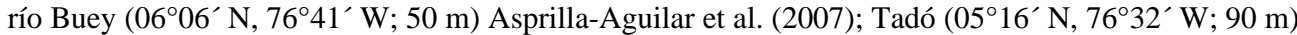
Asprilla-Aguilar et al. (2007); Litoral de San Juan, 2 Km N Playa de Oro, Alto río San Juan (04¹5`32”

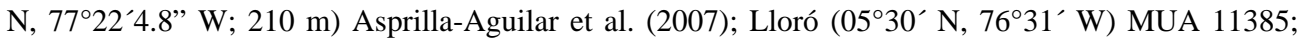
Lloró, Granja experimental de la Universidad Tecnológica del Chocó (05³0`11” N, 76³1‘48” W) ICN 16165*; Pizarro, río Cubarradó, confluencia quebrada Angostura, casa de máquinas (0458` N, 77²2’ W) UV 11338, 11346*; Bajo Baudó Pizarro, corregimiento Piliza, quebrada Señor Señorcito

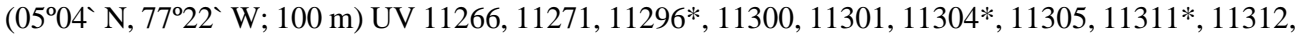
11331; Río Pato, Km 48 vía Panamericana (05³1’33” N, 7659`42” W) UV 3826 - 3828; Chiguero, río

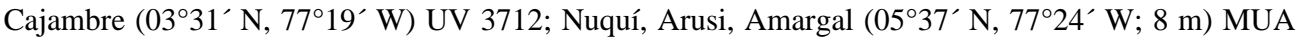
11359. 4. Departamento de Córdoba, Tierralta, vereda La Oscurana, finca Onomas, bosques parte alta finca (0800'14” N, 7605’02” W; 500 m) ICN 19917; 5. Departamento de Risaralda, Pueblo Rico, corregimiento Santa Cecilia, 8 Km aproximados de PLOSAN (05¹7’69” N, 76¹3⒈2” W; 1105 m) ICN 12266*; 6. Departamento del Valle del Cauca, Buenaventura, río Zabaletas, $29 \mathrm{Km}$ SE de Buenaventura (034'80” N, 7657'54” W; 100 m), UV 983, 984, 989, 991,1256; Buenaventura, 10 Km Occidente del poblado de Zabaletas (034' N, 7657’54” W; 100 m); Buenaventura, Base Naval Bahía Málaga $\left(03^{\circ} 55^{\prime} \mathrm{N}, 7^{\circ} 20^{\prime} \mathrm{W}\right)$ UV 5439 - 5446; Buenaventura, corregimiento Bajo Calima, Granja Agroforestal Bajo Calima (0359`46” N, 7658’26.4” W; 60 m) ICN 9399, 9400 - 9405*, UV 2814*, 11630; Buena-

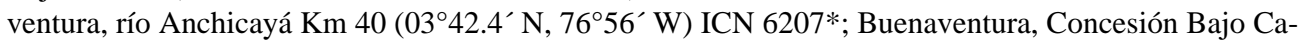
lima, Cuartel B. V. 83 (035' 44” N, 7708’11” W) UV 5777-5780*, 5781, 5783, 5784-5788*, 5791*, 5792*, 5793-5795, 7329, 7330*, 7331*, 10674, 10675, 10676*, 10677*; Buenaventura, Llano Bajo (0344 N, 7657’ W; 50 m) UV 10546*; El Mirador. Subiendo Agua Sucia (Río Cajambre), margen derecha, frente cerro Caja $\left(03^{\circ} 20^{\prime} \mathrm{N}, 7^{\circ} 00^{\prime} \mathrm{W}\right.$; 210 m) ICN 3715. 